\title{
Editorial: Autoimmunity and Chronic Inflammation in Early Life
}

\author{
Ravi Misra ${ }^{1}$, Jennifer Konopa Mulligan ${ }^{2}$, Sarah Rowland-Jones ${ }^{3}$ and Michael Zemlin ${ }^{4}$ \\ ${ }^{1}$ Department of Pediatrics, The University of Rochester Medical Center, Rochester, NY, United States, ${ }^{2}$ Division of \\ Pulmonary, Critical Care \& Sleep Medicine, University of Florida, Gainesville, FL, United States, ${ }^{3}$ Viral Immunology Unit, \\ Nuffield Department of Medicine, Oxford, United Kingdom, ${ }^{4}$ Department for General Pediatrics and Neonatology, Saarland \\ University, Homburg, Germany
}

Keywords: autoimmunity, pediatrics - children, immune development and maturation, early life origin of disease, chronic inflammation

Editorial on the Research Topic

Autoimmunity and Chronic Inflammation in Early Life

\section{INTRODUCTION}

Non-communicable diseases such as cardiovascular diseases, metabolic disease and chronic inflammatory diseases are often attributed to an interplay between genetic predispositions and imprinting mechanisms early in life. In a simplified concept, the fetal development is characterized by the acquisition of immuno-tolerance towards maternal and self-antigens, whereas the neonatal period reflects the acquisition of immune-defense against potentially harmful environmental antigen. Immune development involves a complex cross talk of immune cells in various organs

\section{OPEN ACCESS}

Edited and reviewed by: Betty Diamond,

Feinstein Institute for Medical

Research, United States

*Correspondence: Michael Zemlin michael.zemlin@uks.eu

Specialty section: This article was submitted to Autoimmune and Autoinflammatory Disorders, a section of the journal

Frontiers in Immunology

Received: 19 August 2021 Accepted: 23 August 2021 Published: 09 September 2021

Citation:

Misra R, Mulligan JK, Rowland-Jones $S$ and Zemlin M (2021) Editorial: Autoimmunity and Chronic Inflammation in Early Life.

Front. Immunol. 12:761160. doi: 10.3389/fimmu.2021.761160 that is influenced by environmental antigen (1-3). During infancy and childhood, autoimmune diseases and chronic inflammation coincide with an exponential diversification of the adaptive immune system, causing potentially life-long consequences. Interestingly, the earlier hypothesis of an "immunodeficiency of immaturity" had to be partially revised since it has become clear that inflammatory states in the neonate, e.g. in the context of sepsis, represent a lack of controlling inflammation rather than a failure to mount inflammation. Thus, hyperinflammatory states can occur even in the very immature organism and can lay the ground for autoimmunity or various conditions of chronic inflammation. This knowledge may affect therapeutic approaches.

In this Research Topic, we have called for publications that relate to clinical or molecular aspects of aberrant immune responses in pediatric patients. Here we briefly present the 12 contributions that comprise six Original Research articles, three (mini) reviews and three case reports. The contributions can be grouped into three sections:

1. Clinical manifestations of early autoimmune and chronic inflammatory diseases

2. From molecular mechanisms to autoimmune phenotypes

3. The role of $\mathrm{B}$ - and $\mathrm{T}$ cells for autoimmune diseases and chronic inflammation in early life

\section{CLINICAL MANIFESTATIONS OF EARLY AUTOIMMUNE AND CHRONIC INFLAMMATORY DISEASES}

In a single center overview, Wang et al. present the genetic and clinical characteristics of 79 pediatric patients with monogenetic autoimmune diseases. The patients were affected with 18 different 
diagnoses that can be grouped into inflammasomopathies (42\%), non-inflammasome related conditions (48\%) and type 1 interferonopathies (10\%). $76 \%$ of the patients presented with skin disorders, making this the most common clinical clue to autoimmune diseases. In contrast, Chinello et al. address a new complication in a rare autoimmune disease by presenting a pediatric patient with lipopolysaccharide-responsive-beigelinked-anchor-protein (LRBA) deficiency that developed acute cervical longitudinally transverse myelitis. Chen et al. report a patient with symptoms of intestinal Behcet's disease that was associated with novel heterozygous mutation in the TNFAIP gene, potentially linking intestinal Behcet's disease with Haploinsufficiency A20.

\section{FROM MOLECULAR MECHANISMS TO AUTOIMMUNE PHENOTYPES}

Haploinsufficiency A20 (HA20) has recently been identified as one potential molecular mechanism of autoimmunity. In an analysis of 89 patients with this rare condition, Chen et al. present the typical clinical manifestations associated with HA20: Recurrent oral ulcers and fever episodes, gastrointestinal and genital ulcers as well as skin lesions are the hallmarks that can help clinicians to initiate targeted diagnostics in patients that often suffer a long odyssey prior to a correct diagnosis and therapy. Another diagnostic "chameleon", systemic lupus erythematosus has been addressed by Motwani et al.: In an elegant series of experiments, the authors could rule out a contribution of the cGAS-STING pathway to the clinical symptoms in a murine model of chronic SLE, thus questioning the potential of novel cGAS-STING directed therapeutic approaches. As shown by Wang et al. in this Research Topic, inflammasomopathies represent a major percentage of autoimmune diseases, including type 1 diabetes. In extension of these observations, Sun et al. review the literature on the assembly and function of the NLRP3 inflammasome and its role in type 1 diabetes.

\section{THE ROLE OF B- AND T CELLS FOR AUTOIMMUNE DISEASES AND CHRONIC INFLAMMATION IN EARLY LIFE}

While Sun et al. address the role of the NLRP3 inflammasome in diabetes, Shapiro et al. address $\mathrm{T}$ cell mediated mechanisms in the same disease: The severity of type 1 diabetes was reduced in CD226 deficient non-obese diabetic (NOD) mice. Using adoptive transfer experiments and other techniques, the authors could attribute this phenomenon to the impaired activation of peripheral $\mathrm{T}$ cells, which is mediated by $\mathrm{CD} 226$. Foth et al. contribute a review on the $\mathrm{T}$ cell repertoire during ontogeny and characteristic aberrations in

\section{REFERENCES}

1. Ubags NDJ, Alejandre Alcazar MA, Kallapur SG, Knapp S, Lanone S, Lloyd $\mathrm{CM}$, et al. Early Origins of Lung Disease: Towards an Interdisciplinary Approach. Eur Respir Rev (2020) 29(157):200191. doi: 10.1183/ 16000617.0191-20202 inflammatory disorders. Ultimately, this relates to the unanswered question of whether the $\mathrm{T}$ cell is rather a bystander or a culprit during the development of autoimmunity and chronic inflammatory diseases. The small but still enigmatic subset of IL-17 expressing TH cells plays a key role in the pathogenesis of chronic mucocutaneous candidiasis in children and are diminished in other autoimmune diseases such as hyper IgE syndrome. Shamriz et al. give an overview on the immune mechanisms, current management and novel targeted therapies for monogenic mucocutaneous candidiasis with a focus on TH 17 cells. Two articles of this Research Topic focus on the immunologic and inflammatory aspects of lung disease: Bhattacharya et al. present transcriptomic profiles of purified CD8 $+\mathrm{T}$ cells from preterm neonates at the time of discharge. By identifying signaling pathways that differ between infants with and without bronchopulmonary dysplasia this study sheds new light into the patho-mechanisms of this condition, which remains a major cause of long-term morbidity in preterm neonates. Hwang et al. report that inducible Bronchus-Associated Lymphoid Tissue (iBALT) attenuates the allergic airway inflammation in a mouse model by sequestration of effector T cells. On the other hand, Breville et al. present a case report suggesting a role of IgG4 in severe complement-mediated thrombotic microangiopathy in a patient with IgG4 related disease. Interestingly, a genetic predisposition might enhance the susceptibility to form inhibitory anti-Factor $\mathrm{H}$ IgG4 antibodies.

\section{CONCLUDING REMARKS}

The article collection of the Research Topic gives an up-to-date overview on the clinical manifestations and molecular mechanisms of autoimmunity and chronic inflammation in early life. Thus, in the jungle of "diagnostic chameleons", clinicians may find important hints for linking characteristic symptoms with autoimmune diseases. On the other hand, basic researchers find a thorough overview of the current knowledge on disease mechanism that should inspire research projects in the field of diagnostics and novel therapeutic concepts.

\section{AUTHOR CONTRIBUTIONS}

MZ wrote the first draft of the manuscript. All authors contributed to the article and approved the submitted version.

\section{FUNDING}

This study was supported by BMBF PRIMAL study (01GL1746D) to MZ.

2. Sarkar A, Yoo JY, Valeria Ozorio Dutra S, Morgan KH, Groer M. The Association Between Early-Life Gut Microbiota and Long-Term Health and Diseases. J Clin Med (2021) 10(3):459. doi: 10.3390/jcm 100304593

3. Jian C, Carpén N, Helve O, de Vos WM, Korpela K, Salonen A. Early-Life Gut Microbiota and Its Connection to Metabolic Health in Children: Perspective on 
Ecological Drivers and Need for Quantitative Approach. EBioMedicine (2021) 69:103475. doi 10.1016/j.ebiom.2021.103475

Conflict of Interest: The authors declare that the research was conducted in the absence of any commercial or financial relationships that could be construed as a potential conflict of interest.

Publisher's Note: All claims expressed in this article are solely those of the authors and do not necessarily represent those of their affiliated organizations, or those of the publisher, the editors and the reviewers. Any product that may be evaluated in this article, or claim that may be made by its manufacturer, is not guaranteed or endorsed by the publisher.

Copyright $\odot 2021$ Misra, Mulligan, Rowland-Jones and Zemlin. This is an openaccess article distributed under the terms of the Creative Commons Attribution License (CC BY). The use, distribution or reproduction in other forums is permitted, provided the original author(s) and the copyright owner(s) are credited and that the original publication in this journal is cited, in accordance with accepted academic practice. No use, distribution or reproduction is permitted which does not comply with these terms. 